\title{
Long-range odd triplet superconductivity in SF structures with Néel walls
}

\author{
A. F. Volkov, ${ }^{1,2, \text { 田 Ya. V. Fominov, }}$, 团 and K. B. Efetov ${ }^{1,3}$ \\ ${ }^{1}$ Theoretische Physik III, Ruhr-Universität Bochum, D-44780 Bochum, Germany \\ ${ }^{2}$ Institute of Radioengineering and Electronics of the Russian Academy of Sciences, 103907 Moscow, Russia \\ ${ }^{3}$ L. D. Landau Institute for Theoretical Physics RAS, 119334 Moscow, Russia
}

(Dated: 27 October 2005)

\begin{abstract}
We consider a multidomain superconductor/ferromagnet (SF) structure with an in-plane magnetization, assuming that the neighboring domains are separated by the Néel domain walls. We show that an odd triplet long-range component arises in the domain walls and spreads into domains over a long distance of the order $\xi_{T}=\sqrt{D / 2 \pi T}$ (in the dirty limit). The density of states variation in the domains due to this component changes over distances of the order $\xi_{T}$ and turns to zero in the middle of domains if the magnetization rotates in the same direction in all domain walls.
\end{abstract}

PACS numbers: 74.45.+c, 74.78.Fk, 74.50.+r, 75.70.Kw

\section{INTRODUCTION}

The past decade has seen a rapid growth of interest in the study of hybrid superconductor-ferromagnet (SF) structures (see, for example, reviews 1, 2.3). The interest in such systems originates from the possibility of finding new physical phenomena as well from the hope of constructing new devices based on these structures. New physical phenomena arising in these systems are the result of a nontrivial interplay of competing types of ordering in superconductors and ferromagnets. Superconducting correlations lead in superconductors to the appearance of the Cooper pairs, that is, the pairs of electrons with opposite spins. On the opposite, the exchange interaction in ferromagnets tries to align the electron spins in one direction. In SF structures these two types of interactions are spatially separated and can coexist despite much greater value of the exchange energy $h$ in comparison to the superconducting order parameter $\Delta$.

Due to the proximity effect ${ }^{4}$ the superconducting correlations penetrate the ferromagnet in SF structures. The opposite effect, i.e., the penetration of a magnetic moment $\mathbf{M}$ into the superconductor, also takes place. It turns out that the magnetic moment $\mathbf{M}_{S}$ is induced in the superconductor. The magnetic moment $\mathbf{M}_{S}$ is aligned in the direction opposite to the magnetization direction of free electrons in the ferromagnet and spreads over a distance of the order of the superconducting correlation length $\xi_{S}=\sqrt{D_{S} / \Delta}$ (in the dirty limit) 5 On the other hand the condensate wave function $f$ penetrates the ferromagnet with an uniform magnetization $\mathbf{M}_{F}$ over a much shorter distance of the order of the "exchange length" $\xi_{h}=\sqrt{D_{F} / h} .1 .2$ The condensate wave function decays in $\mathrm{F}$ in a nonmonotonic way as $f(x) \sim \exp \left(-x / \xi_{h}\right) \cos \left(x / \xi_{h}\right)$; it oscillates in space and decreases exponentially. This nonmonotonic behavior of $f(x)$ leads to a nonmonotonic dependence of the critical temperature $T_{c}$ of the superconducting transition in SF bilayers and multilayers 1 2.6.7.8.9.10 and to a $\pi$-state in SFS Josephson junctions, 1,2,11,12,13,14,15,16

In the case of a nonuniform magnetization in the ferro- magnet a new phenomenon appears: a triplet component of the condensate wave function $f$ (generally speaking the condensate wave function is a matrix in the particle-hole and spin space) arises in the SF system ${ }^{3}$ This triplet component is an odd function of the Matsubara frequency $\omega$ (while the conventional BCS singlet component of $f$ is an even function of $\omega$ ) and spreads in the ferromagnet over a long distance of the order of $\xi_{T}=\sqrt{D_{F} / 2 \pi T}$. This long-range triplet odd-frequency component was predicted to exist in a SF structure with nonhomogeneous magnetization in Ref. 17 and this prediction was confirmed for a slightly different case in Ref. 18. In Refs. 17 and 18, a SF structure with a domain wall at the SF interface was considered, that is, it was assumed that $\mathbf{M}_{F}=M(0, \sin \alpha(x), \cos \alpha(x))$, where $\alpha(x)=Q x$ in the interval $0<x<a_{Q}$ and $\alpha(x)=Q a_{Q}$ at $x>a_{Q}$. The triplet component $f_{L}$ was shown to arise in the domain wall and to penetrate the ferromagnet over a long distance $\xi_{T}$. Unlike the triplet component in superfluid ${ }^{3} \mathrm{He}$ and in $\mathrm{Sr}_{2} \mathrm{RuO}_{4}$, this odd triplet component corresponds to $s$-wave correlations and hence is symmetric in the momentum space; therefore it is not destroyed by scattering on ordinary, nonmagnetic impurities, and survives in the dirty limit. We call this component the long-range triplet component (LRTC). The LRTC may also arise in a SF structure with a uniform magnetization and spin-active interface 19 Note that from a macroscopic point of view a domain wall at the SF interface can also be considered as a "spin-active interface". The LRTC may arise in a multilayered SF structure with noncollinear orientations of the magnetization vector $\mathbf{M}_{F_{i}}$ in different $\mathrm{F}_{i}$ layers ${ }^{20.21}$ In particular, a new type of superconductivity (odd triplet superconductivity) has been predicted in such structures if the thickness of the $\mathrm{F}$ layers $d$ obeys the condition: $\xi_{h} \ll d \lesssim \xi_{T}$. In this case the Josephson coupling between neighboring $\mathrm{F}$ layers is realized only via the LRTC because the singlet component decays very fast in the $\mathrm{F}$ layers. Therefore superconductivity in the transverse direction is due to the LRTC, whereas in-plane superconductivity is caused mainly by the singlet BCS component. The influence of the LRTC on the critical temperature of the superconducting transition in FSF 
structures with a noncollinear magnetization orientation was studied in Ref. 22 .

Historically, the odd-frequency triplet pairing was conjectured in 1974 by Berezinsii 23 as a possible mechanism for superfluidity in ${ }^{3} \mathrm{He}$. It turned out later that in ${ }^{3} \mathrm{He}$ another type of triplet pairing (even in frequency, odd in momentum) is realized. Odd-frequency pairing in solids was also studied in Refs. 24.25 26. A triplet odd-frequency pairing was investigated in Refs. 24 (twodimensional electron gas with repulsion in the presence of impurities) and 25 (a Kondo lattice model). A singlet odd-frequency pairing was analyzed 26 as a possible type of pairing in high-temperature superconductors.

Although several experimental results may be interpreted in terms of the LRTC, 27.28.29.30.31.32 there are still no direct experimental evidences in favor of the odd triplet superconductivity. Therefore there is a need to investigate, in more detail, a possibility to observe this new type of superconductivity. One of the important and inherent features of ferromagnets is the domain structure. The domain structure in a ferromagnet may essentially alter the properties of SF structures. For example, modulation of the phase difference $\varphi$ between two superconductors due to internal magnetic fields in the ferromagnetic domains, can lead to a negative critical current $I_{c}$ in the Josephson SFS junction ${ }^{33}$ The influence of domains on $I_{c}$ in SFS Josephson junctions was studied in Refs. 34 and 35. In Ref. 34 the magnetization vector is assumed to be in-plane and to rotate around the direction normal to the plane of the junction. In this case the LRTC arises, and the possible $\pi$-state may be suppressed due to an effective averaging of the exchange field. In Ref. 35 a SFS junction with two in-plane domains of opposite orientation was studied (no LRTC arises in this case). It was shown that if the thicknesses of the domains are equal, the critical current $I_{c}$ is always positive.

In realistic domain structures, the domains are separated by domain walls. Below we shall discuss the case when the magnetization vector $\mathbf{M}_{F}$ lies in the plane of the $\mathrm{F}$ film and the domain walls are of the Néel type. ${ }^{36}$ A limiting case of such a structure was considered in Refs. 37 and 38. It was assumed that the vector $M_{F}$ rotates in space continuously

$$
\mathbf{M}_{F}(y)=M_{0}(0, \sin Q y, \cos Q y)
$$

(we choose the $x$ axis normal to the plane of the $\mathrm{F}$ film, whereas in Ref. 38 the $z$ axis is normal to the plane of the F film). The possibility of a cryptoferromagnetic state in SF structures with the magnetization $M_{F}(y)$ given by Eq. (1) was studied in Ref. 37. The F layer was supposed to be very thin: $d \ll \xi_{h}$. A solution for the Eilenberger equation has been found near the critical temperature $T_{c}$ of the superconducting transition. It was established that in a certain interval of parameters $(Q, d$, etc.) a homogeneous state in the ferromagnet becomes energetically unfavorable and the nonhomogeneous magnetization determined by Eq. (1) arises in the F film. In Ref. 38 a SF bilayer with the F film of arbitrary thickness was
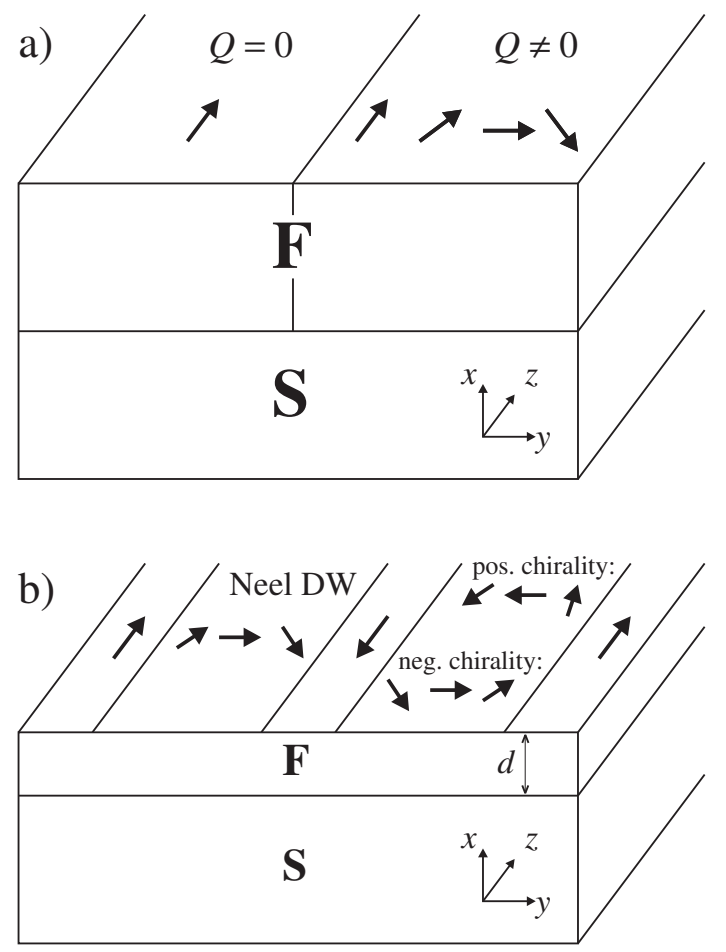

FIG. 1: SF systems considered in the paper. (a) SF bilayer with half-infinite $\mathrm{S}$ and $\mathrm{F}$ parts. The domain $(y<0)$ and the region with rotating magnetization $(y>0)$ are also halfinfinite. (b) Multidomain F layer of thickness $d$ in contact with a bulk superconductor. Depending on the relative orientation of rotating magnetizations in neighboring domain walls, we distinguish the cases of positive and negative chirality ( $Q$ has the same or opposite sign in the neighboring domain walls, respectively). The proportion between the widths of domains and domain walls is chosen only for drawing purposes.

studied in the dirty limit. Analysis of a solution for the Usadel equation shows that in the case of magnetization, uniformly rotating along the F film [see Eq. (1)], the condensate function $f$ penetrates into the $\mathrm{F}$ film over a short distance of the order $\xi_{h}$. The LRTC is absent in this case. According to Refs. 17 18.19 21, the LRTC appears if $\mathbf{M}_{F}$ rotates across the $\mathrm{F}$ layer (i.e., depends not on $y$ but on $x$ ).

In the present paper we consider a domain structure in a thin F film, where domains with antiparallel in-plane magnetizations are separated by the Néel walls (while the magnetization does not change across the thin $\mathrm{F}$ film). This domain structure is realized in real ferromagnetic films ${ }^{36}$ The $y z$ plane is chosen to be parallel to the $\mathrm{SF}$ interface (see Fig. 1). We show that the LRTC arises at the Néel domain walls and decays exponentially away from the domain walls and the SF interface over a long distance $\xi_{T}$. We calculate the density of states (DOS) variation $\delta \nu$ in the ferromagnet caused by the proximity effect and find that $\delta \nu(y)$ turns to zero in the middle of domains in the case of positive chirality.

The paper is organized as follows. In Sec. II we formu- 
late the Usadel equations, the corresponding boundary conditions, and investigate the main features of the longrange triplet superconducting component which appears due to the presence of Néel domain walls. The analysis is made on the simplified model with half-infinite $\mathrm{S}$ and $\mathrm{F}$ layers and only one half-infinite region with rotating magnetization. In Sec. III we consider the case of the multidomain F layer, employing the results of Sec. III To make the model realistic, in Sec. IV we take into account a finite thickness of the ferromagnet. To illustrate the results for the LRTC, we study the density of states due to it. Finally, we present our conclusions in Sec. $\square$

\section{BASIC EQUATIONS. HALF-INFINITE DOMAIN}

Consider a ferromagnet $(x>0)$ in contact with a superconductor $(x<0)$. We assume that the in-plane exchange field $\mathbf{h}(y)$ in the $\mathrm{F}$ layer, which is proportional to the magnetization $\mathbf{M}_{F}$, depends on $y: \mathbf{h}(y)=h(0,0,1)$ at $y<0$ and $\mathbf{h}(y)=h(0, \sin \alpha(y), \cos \alpha(y))$ with $\alpha(y)=$ $Q y$ at $y>0$. This means that the magnetization vector $\mathbf{M}_{F}$ is oriented along the $z$ axis at $y<0$ and rotates in the $y z$ plane at $y>0$. The region with rotating magnetization models a Néel domain wall. The structure that we discuss first is shown in Fig. 1(a) and contains only one half-infinite domain and one half-infinite region with rotating magnetization; the thickness of the ferromagnet is also infinite. Then we shall use the obtained results to describe a realistic structure depicted in Fig. 1(b).

Our goal is to find the condensate Green functions in the ferromagnet, induced due to the proximity effect. We consider the dirty limit, which means, in particular, that $h \tau \ll 1$, where $\tau$ is the momentum relaxation time due to elastic scattering.

We employ a widely used model: an exchange field $h$ acts on free electrons and there is no attractive interaction in the $\mathrm{F}$ layer leading to the superconducting order parameter ${ }^{1.2 .3}$ However, the condensate (Gor'kov) functions are finite in the $\mathrm{F}$ region due to the boundary conditions at the SF interface. We are interested in distances much larger than the Fermi wavelength, therefore we can use the quasiclassical Green functions $\check{g}^{R(A)}$. The matrix retarded (advanced) functions $\check{g}^{R(A)}$ in the ferromagnet obey the Usadel equation ${ }^{3}$

$$
D \nabla\left(\check{g}^{R(A)} \nabla \check{g}^{R(A)}\right)+i \varepsilon\left[\hat{\tau}_{3}, \check{g}^{R(A)}\right]-i\left[\check{h}, \check{g}^{R(A)}\right]=0,
$$

where $\varepsilon$ is energy, $\check{h}=h\left(\hat{\tau}_{3} \hat{\sigma}_{3} \cos \alpha(y)+\hat{\tau}_{0} \hat{\sigma}_{2} \sin \alpha(y)\right)$, and $\alpha(y)=0$ at $y \leqslant 0$ while $\alpha(y)=Q y$ at $y \geqslant 0$. We assume that the diffusion constants $D$ for electrons with spin up and down are equal (this is correct if the exchange energy $h$ is much less than the Fermi energy $\left.\varepsilon_{F}\right)$. The matrix Green functions $\check{g}$ are $4 \times 4$ matrices in the Gor'kov-Nambu and spin spaces. $\hat{\tau}_{i}$ and $\hat{\sigma}_{i}$ are the Pauli matrices in the Gor'kov-Nambu and spin spaces, respectively.
We represent $\check{g}^{R(A)}$ in the form

$$
\check{g}^{R(A)}= \pm \hat{\tau}_{3} \hat{\sigma}_{0}+\check{f},
$$

where the first term is the retarded (advanced) quasiclassical Green function in the normal state. The superconducting correlations, described by $\breve{f}(x, y)$, are assumed to be weak due to the finite interface transparency (resulting from an oxide barrier or from mismatch in the Fermi surfaces of $\mathrm{S}$ and $\mathrm{F}$ materials). In the considered case of weak proximity effect $(|\breve{f}| \ll 1)$, Eq. (2) can be linearized. In the Matsubara representation it acquires the form

$$
\begin{aligned}
\nabla^{2} \check{f}-2 k_{\omega}^{2} \check{f}-i k_{h}^{2} \operatorname{sgn} \omega & \left(\left\{\hat{\sigma}_{3}, \check{f}\right\} \cos \alpha(y)\right. \\
+ & \left.\hat{\tau}_{3}\left[\hat{\sigma}_{2}, \check{f}\right] \sin \alpha(y)\right)=0,
\end{aligned}
$$

where $\omega=\pi T(2 n+1), k_{\omega}^{2}=|\omega| / D, k_{h}^{2}=h / D$, the square brackets denote the commutator, and the braces denote the anticommutator.

The Green function in the bulk of the superconductor is $\check{f}_{S}=\hat{\tau}_{2} \hat{\sigma}_{3} f_{S}$, with $f_{S}=\Delta / \sqrt{\omega^{2}+\Delta^{2}}$. We shall use the following boundary condition for $\check{f}$ at the SF interface $(x=0)$ :

$$
\frac{\partial \check{f}}{\partial x}=-\frac{\check{f}_{S}}{\gamma_{b}},
$$

where $\gamma_{b}=R_{b} \sigma$, while $\sigma$ is the conductivity of the ferromagnet and $R_{b}$ is the interface resistance per unit area. This boundary condition follows from the general ones ${ }^{39.40}$ if two assumptions are made: (1) the proximity effect is weak (i.e., $\gamma_{b} / \xi_{h} \gg 1$ ) and (2) the bulk solution $\check{f}_{S}$ in the superconductor is unperturbed and valid up to the interface (i.e., $\gamma_{b} / \xi_{S} \gg \sigma / \sigma_{S}$ ).

We can rewrite Eq. (4) in the form taking into account boundary condition (5):

$$
\begin{aligned}
\frac{\partial^{2} \check{f}}{\partial x^{2}}+\frac{\partial^{2} \check{f}}{\partial y^{2}}- & 2 k_{\omega}^{2} \check{f}-i k_{h}^{2} \operatorname{sgn} \omega\left(\left\{\hat{\sigma}_{3}, \check{f}\right\} \cos \alpha(y)\right. \\
& \left.+\hat{\tau}_{3}\left[\hat{\sigma}_{2}, \check{f}\right] \sin \alpha(y)\right)=-\frac{2 \check{f}_{S}}{\gamma_{b}} \delta(x) .
\end{aligned}
$$

We must seek for an even solution of this equation, then this is equivalent to the problem with the boundary condition (we have reflected $\check{f}$ with respect to $x=0$ and now solve the equation at all $x$ ). However, the requirement that the solution is even, will be automatically satisfied: as we shall see below, the Fourier harmonics (over $x$ ) depend only on $k^{2}$, hence they are even in $k$, which means that $f(x)$ is even in $x$.

Performing the Fourier transformation $\check{f}(k, y)=$ $\int d x \check{f}(x, y) \exp (-i k x)$, we obtain

$$
\begin{aligned}
\frac{\partial^{2} \check{f}}{\partial y^{2}}-\left(k^{2}+2 k_{\omega}^{2}\right) \check{f} & -i k_{h}^{2} \operatorname{sgn} \omega\left(\left\{\hat{\sigma}_{3}, \check{f}\right\} \cos \alpha(y)\right. \\
& \left.+\hat{\tau}_{3}\left[\hat{\sigma}_{2}, \check{f}\right] \sin \alpha(y)\right)=-\frac{2 \check{f}_{S}}{\gamma_{b}} .
\end{aligned}
$$


At $y>0$ the function $\alpha(y)$ is $y$-dependent, while at $y<0$ we have $\alpha=0$. In the region of positive $y$ one can exclude the $y$-dependence from Eq. (7) with the aid of rotation

$$
\check{f}=\check{U} \check{f}_{u} \check{U}^{+}
$$

where $\check{U}=\exp \left(i \hat{\tau}_{3} \hat{\sigma}_{1} \alpha(y) / 2\right)$. As a result, we get $(y>0)$

$$
\begin{aligned}
& \frac{\partial^{2} \check{f}_{u}}{\partial y^{2}}-\left(k^{2}+\frac{Q^{2}}{2}+2 k_{\omega}^{2}\right) \check{f}_{u}-\frac{Q^{2}}{2} \hat{\sigma}_{1} \check{f}_{u} \hat{\sigma}_{1} \\
& \quad+i Q \hat{\tau}_{3}\left\{\hat{\sigma}_{1}, \frac{\partial \check{f}_{u}}{\partial y}\right\}-i k_{h}^{2} \operatorname{sgn} \omega\left\{\hat{\sigma}_{3}, \check{f}_{u}\right\}=-\frac{2 \check{f}_{S}}{\gamma_{b}}
\end{aligned}
$$

in terms of the new function $\check{f}_{u}(k, y)$. The same equation is valid for $y<0$ if we set $Q=0$ :

$$
\frac{\partial^{2} \check{f}_{u}}{\partial y^{2}}-\left(k^{2}+2 k_{\omega}^{2}\right) \check{f}_{u}-i k_{h}^{2} \operatorname{sgn} \omega\left\{\hat{\sigma}_{3}, \check{f}_{u}\right\}=-\frac{2 \check{f}_{S}}{\gamma_{b}} .
$$

The original functions $\check{f}$ and $\partial \check{f} / \partial y$ are continuous at $y=0$. Therefore the rotated functions obey the following boundary conditions at $y=0$ :

$$
\begin{aligned}
\check{f}_{u}(-0) & =\check{f}_{u}(+0), \\
\frac{\partial \check{f}_{u}(-0)}{\partial y} & =\frac{\partial \check{f}_{u}(+0)}{\partial y}+i \frac{Q}{2} \hat{\tau}_{3}\left\{\hat{\sigma}_{1}, \check{f}_{u}\right\} .
\end{aligned}
$$

Thus we have to solve the linear matrix Eqs. (9) $(y>$ $0)$ and (10) $(y<0)$ of the second order with the boundary conditions (11) and (12) at $y=0$. We can represent the solution in the form

$$
\check{f}_{u}=\check{F}(Q) \theta(y)+\check{F}(0) \theta(-y)+\delta \check{f}_{u},
$$

where $\theta$ is the Heaviside step function and the constants $\check{F}(Q)$ and $\check{F}(0)$ are the homogeneous solutions of Eqs. (9) and (10) at $y= \pm \infty$. The matrices $\check{F}$ have the form

$$
\check{F}=\hat{\tau}_{2}\left(\hat{\sigma}_{0} F_{0}+\hat{\sigma}_{3} F_{3}\right)
$$

where

$$
\begin{aligned}
& F_{0}(Q)=-\frac{4 i f_{S} k_{h}^{2} \operatorname{sgn} \omega}{\gamma_{b} \mathcal{D}(Q)}, \\
& F_{3}(Q)=\frac{2 f_{S}\left(k^{2}+Q^{2}+2 k_{\omega}^{2}\right)}{\gamma_{b} \mathcal{D}(Q)},
\end{aligned}
$$

and

$$
\mathcal{D}(Q)=\left(k^{2}+Q^{2}+2 k_{\omega}^{2}\right)\left(k^{2}+2 k_{\omega}^{2}\right)+4 k_{h}^{4} .
$$

The correction $\delta \check{f}_{u}(k, y)$ obeys the same Eqs. (9) and (10) without the right-hand side. It has the form

$$
\delta \check{f}_{u}=\hat{\tau}_{2} \hat{\sigma}_{3} f_{3}+\hat{\tau}_{2} \hat{\sigma}_{0} f_{0}+\hat{\tau}_{1} \hat{\sigma}_{1} f_{1}
$$

The first term is the singlet component. The second term is the triplet component with zero projection of the
Cooper pair spin on the $z$ axis. This component arises even in the case of a homogenous magnetization of the ferromagnet and decays in the $\mathrm{F}$ film over the short distance $\xi_{h}$. The last term in Eq. (18) is the triplet component with the spin moment projection \pm 1 . It arises in the case of a nonhomogeneous magnetization and decays over a long distance of the order $\xi_{T}$. The functions $f_{i}(k, y)$ in Eq. (18) can be represented as a sum of eigenfunctions of Eqs. (9) and (10), i.e.,

$$
\begin{aligned}
& f_{i}(y)=\sum_{l} A_{i l} \exp \left(-\kappa_{l}(Q) y\right), \quad \text { at } y>0 \\
& f_{i}(y)=\sum_{l} B_{i l} \exp \left(\kappa_{l}(0) y\right), \quad \text { at } y<0 .
\end{aligned}
$$

The inverse decay lengths $\kappa_{l}(Q)$ are the eigenvalues of Eqs. (9) and (10) (without the right-hand side). The equation for $\kappa_{l}(Q)$ has the form $(l=1,2,3)$

$$
\begin{gathered}
{\left[\left(\kappa_{l}^{2}-k^{2}-Q^{2}-2 k_{\omega}^{2}\right)^{2}+4\left(Q \kappa_{l}\right)^{2}\right]\left(\kappa_{l}^{2}-k^{2}-2 k_{\omega}^{2}\right)} \\
+4 k_{h}^{4}\left(\kappa_{l}^{2}-k^{2}-Q^{2}-2 k_{\omega}^{2}\right)=0
\end{gathered}
$$

We assume that the exchange length is the shortest length in the problem:

$$
k_{h}^{2} \gg k^{2}, Q^{2}, k_{\omega}^{2}
$$

Then the eigenvalues $\kappa_{l}$ consist of two "short-range" values

$$
\kappa_{ \pm} \approx(1 \mp i \operatorname{sgn} \omega) k_{h}
$$

and one "long-range" value

$$
\kappa_{L}(Q) \approx \sqrt{k^{2}+Q^{2}+2 k_{\omega}^{2}}
$$

At $y<0$ we have the same $\kappa_{l}$ with $Q=0$.

Calculating the corresponding eigenvectors under assumption (22), in the first order over $1 / k_{h}$ we obtain

$$
A_{0 \pm} \approx \mp A_{3 \pm}, \quad A_{1 \pm} \approx \mp \frac{2 Q}{\kappa_{ \pm}} A_{3 \pm}, \quad A_{3,0 L} \approx 0 .
$$

To be more exact, $A_{3 L} \approx\left(Q \kappa_{L} / i k_{h}^{2} \operatorname{sgn} \omega\right) A_{1 L}$ and $A_{0 L}$ is even smaller. The same relations with $Q=0$ hold for $B_{i l}$, which yields

$$
B_{0 \pm} \approx \mp B_{3 \pm}
$$

for nonzero coefficients.

The next step is to match solutions (19) and (20) with the help of boundary conditions (11) and (12) and to find the coefficients $A_{i l}$ and $B_{i l}$. This simple but cumbersome calculation is presented in the Appendix. In the considered limit of a small exchange length [see Eq. [22)], the coefficients $A_{1 L} \approx B_{1 L}$ [see Eq. A.15] that describe the LRTC are the largest ones. In this limit the function 
$F_{0}(Q)$ has a simple form (A.12). Therefore the magnitude of the LRTC at the interface between a domain and a domain wall $(y=0)$ is equal to

$$
f_{L}(k, 0) \equiv f_{1}(k, 0)=-\frac{i f_{S} \operatorname{sgn} \omega}{\gamma_{b} k_{h}^{2}} \frac{Q}{\kappa_{Q}+\kappa_{0}},
$$

where for brevity we have denoted

$$
\kappa_{Q} \equiv \kappa_{L}(Q), \quad \kappa_{0} \equiv \kappa_{L}(0)
$$

Now we return to the real space and analyze our results. The spatial dependence of the LRTC at $y<0$ is [see Eq. (20)]

$$
f_{L}(x, y)=\int \frac{d k}{2 \pi} f_{L}(k, 0) \exp (i k x) \exp \left(\kappa_{0} y\right) .
$$

From this formula one can easily find the asymptotic behavior of the LRTC $f_{L}(x, y)$. For large negative $y$ $\left(|y| \gg 1 / k_{\omega}\right)$, we expand $\kappa_{0}$ and $\kappa_{Q}$ with respect to $\left(k / k_{\omega}\right)^{2}$ (since the characteristic $k$ in the integral is of the order $\left.\sqrt{k_{\omega} /|y|}\right)$ and obtain

$$
\begin{aligned}
f_{L}(x, y) & =-\frac{i f_{S} Q \operatorname{sgn} \omega}{\gamma_{b} k_{h}^{2}} \sqrt{\frac{k_{\omega}}{\sqrt{2} \pi|y|}} \\
& \times \frac{\exp \left(-\sqrt{2} k_{\omega}|y|\right) \exp \left(-x^{2} k_{\omega} / \sqrt{2}|y|\right)}{\sqrt{2} k_{\omega}+\sqrt{2 k_{\omega}^{2}+Q^{2}}} .
\end{aligned}
$$

This formula shows that the condensate function $f_{L}(x, y)$ decays exponentially with increasing $y$, but the characteristic length is rather large $\left(\sim k_{\omega}^{-1}\right)$.

For comparison, we can calculate the short-range component at the SF interface in the case of a homogenous magnetization: $f_{0}=-i f_{S} \operatorname{sgn} \omega / 2 \gamma_{b} k_{h}$ [it follows directly from Eq. (10)]. Formula (30) shows that at the SF interface $(x=0)$ and at distances $|y| \lesssim k_{\omega}^{-1}$, the function $f_{L}(x, y)$ is of the order $f_{S} \min \left(Q, k_{\omega}\right) / \gamma_{b} k_{h}^{2}$, which is smaller than the amplitude of the short-range component by the parameter $\min \left(Q, k_{\omega}\right) / k_{h}$. Thus, the interface amplitude of the LRTC is smaller, however it decays much slower in space.

In the domain wall $(y>0)$ the behavior of the function $f_{L}(x, y)$ is nearly the same as at $y<0$ [Eq. (30)] if $Q<$ $k_{\omega}$. In the opposite limit $Q>k_{\omega}$ the function $f_{L}(x, y)$ in the domain wall decays faster: $f_{L}(x, y) \propto \exp (-Q|y|)$.

Having found the condensate function $\check{f}$, we can calculate the density of states (DOS) in the ferromagnetic region. The DOS, normalized to the normal-metallic value, is given by the general formula

$$
\nu(\varepsilon)=\left.\frac{1}{4} \operatorname{Re} \operatorname{Tr}\left(\hat{\tau}_{3} \hat{\sigma}_{0} \check{g}\right)\right|_{\omega \rightarrow-i \varepsilon} .
$$

Using the normalization condition $\check{g}^{2}=1$ and the smallness of the condensate function, we can write the correction to the DOS due to the proximity effect as

$$
\delta \nu(\varepsilon)=-\left.\frac{\operatorname{Re} f_{L}^{2}}{2}\right|_{\omega \rightarrow-i \varepsilon}
$$

(we consider the region in space where only the LRTC is essential). This expression is valid at any $Q$, both zero and nonzero. Equations (30) and (32) show that the LRTC changes the DOS in the ferromagnet at distances much larger than the exchange length $\xi_{h}$.

\section{TRIPLET COMPONENT IN MULTIDOMAIN SF STRUCTURES}

In this section we study the LRTC in a SF structure with a multidomain ferromagnetic layer, still assuming infinite thickness of the F layer [Fig. 1(b) with $d \rightarrow \infty$ ]. One can distinguish between two possibilities: (a) positive chirality, when the magnetization vector $\mathbf{M}(y)$ in all the domain walls rotates in the same direction (e.g., clockwise), and (b) negative chirality, when the vector $\mathbf{M}(y)$ in neighboring domain walls rotates in the opposite directions [e.g., clockwise in the $2 n$-th domain walls and counterclockwise in the $(2 n+1)$-th domain walls]. We are interested in the LRTC assuming that the exchange length $\xi_{h}$ is much smaller than the coherence length $\xi_{T}$. At distances $x$ essentially exceeding the length $\xi_{h}$ only the LRTC survives in the F layer.

We assume that the width of the domains with $Q=0$ is $2 a_{0}$ and the width of the domain walls $(Q \neq 0)$ is $2 a_{Q}$. The origin $(y=0)$ is located in the middle of a domain with the constant magnetization. At $x \gg \xi_{h}$ only the long-range components of the condensate function survive in the ferromagnet. The largest long-range component is the LRTC. At the boundary between a domain and a domain wall the solution must satisfy boundary conditions (11) and (12). Consider first the case of positive chirality. The angle $\alpha(y)$ is then an odd function of $y$, which means that $f_{1}(y)$ is also odd - this general symmetry can be demonstrated in Eq. (6). Hence the solution for the LRTC is

$$
\begin{aligned}
& f_{1}(y)=A \sinh \left(\kappa_{0} y\right), \quad-a_{0}<y<a_{0}, \\
& f_{1}(y)=B \sinh \left(\kappa_{Q}\left(y-a_{0}-a_{Q}\right)\right), \quad a_{0}<y<a_{0}+2 a_{Q} .
\end{aligned}
$$

Matching these solutions and their derivatives at $y=a_{0}$, we find

$$
B=-A \frac{\sinh \theta_{0}}{\sinh \theta_{Q}}=-\frac{Q F_{0}}{\cosh \theta_{Q}\left(\kappa_{Q}+\kappa_{0} \frac{\tanh \theta_{Q}}{\tanh \theta_{0}}\right)},
$$

where $\theta_{Q}=\kappa_{Q} a_{Q}$ and $\theta_{0}=\kappa_{0} a_{0}$. The amplitude of the LRTC at $y=a_{0}$ is

$$
f_{1}\left(a_{0}\right)=\frac{Q F_{0}}{\kappa_{Q} \operatorname{coth} \theta_{Q}+\kappa_{0} \operatorname{coth} \theta_{0}} .
$$

We see that $f_{1}\left(a_{0}\right)$ turns to zero both at $a_{Q} \rightarrow 0$ and $a_{0} \rightarrow 0$. These limits mean that the widths of the domain walls and domains are assumed to be small in comparison with $\xi_{T}$ while larger than $\xi_{h}$. The case $a_{Q}=0$ implies 
that we have a domain structure with collinear magnetization orientation. The case $a_{0}=0$ corresponds to a SF structure with continuously rotating magnetization (the case studied in Ref. 38). In both cases, the LRTC does not arise.

The spatial dependence of the LRTC in the domain $\left(|y|<a_{0}\right)$, corresponding to Eq. (36), is given by the inverse Fourier transformation

$$
f_{L}(x, y)=\int \frac{d k}{2 \pi} e^{i k x} f_{1}\left(a_{0}\right) \frac{\sinh \left(\kappa_{0} y\right)}{\sinh \theta_{0}} .
$$

Interestingly, the function $f_{L}(x, y)$ turns to zero in the center of a domain $(y=0)$. This means that the DOS variation due to the LRTC also turns to zero in the domain center.

Consider now the case of negative chirality, when the $\mathbf{M}$ vector rotates in the opposite directions in neighboring domain walls. In this case the spatial dependence of the function $f_{1}(y)$ in domain walls remains the same as before, i.e., this function is an odd function with respect to the center of a domain wall. However the spatial dependence of the LRTC in domains changes drastically: it becomes an even function with respect to the center of a domain. Therefore this dependence is

$$
\begin{aligned}
& f_{1}(y)=C \cosh \left(\kappa_{0} y\right), \quad-a_{0}<y<a_{0}, \\
& f_{1}(y)=D \sinh \left(\kappa_{Q}\left(y-a_{0}-a_{Q}\right)\right), \quad a_{0}<y<a_{0}+2 a_{Q} .
\end{aligned}
$$

From boundary conditions (11) and (12) we find the coefficients $C$ and $D$, and finally

$$
f_{1}\left(a_{0}\right)=\frac{Q F_{0}}{\kappa_{Q} \operatorname{coth} \theta_{Q}+\kappa_{0} \tanh \theta_{0}} .
$$

In this case the LRTC disappears only in the limit $a_{Q} \rightarrow 0$ because in this limit one again has a domain structure with collinear magnetization orientation and very narrow domain walls.

Another type of SF structures, sensitive to the chirality of the vector $\mathbf{M}$, was considered in Refs. 20 and 21. It was shown that the sign of the critical Josephson current in a multilayered SF structure depends on chirality.

\section{FINITE THICKNESS OF MULTIDOMAIN F LAYER}

In this section we consider a realistic structure with a ferromagnetic layer of finite thickness $d$, see Fig. 1(b). We again have to solve Eq. (4) with boundary conditions. The first of them, at the SF interface, is Eq. (5) and the other one, at the free surface of the ferromagnetic layer, is

$$
\left.\frac{\partial \check{f}}{\partial x}\right|_{x=d}=0 .
$$

Similarly to Sec. III we can continue $\check{f}$ to the whole $x$ axis, reflecting it with respect to $x=0$ and periodically continuing from the $(-d, d)$ interval. The advantage of this trick is that the boundary conditions are included into the equation. Similarly to Eq. (6), we obtain

$$
\begin{aligned}
& \frac{\partial^{2} \check{f}}{\partial x^{2}}+\frac{\partial^{2} \check{f}}{\partial y^{2}}-2 k_{\omega}^{2} \check{f}-i k_{h}^{2} \operatorname{sgn} \omega\left(\left\{\hat{\sigma}_{3}, \check{f}\right\} \cos \alpha(y)\right. \\
& \left.+\hat{\tau}_{3}\left[\hat{\sigma}_{2}, \check{f}\right] \sin \alpha(y)\right)=-\frac{2 \check{f}_{S}}{\gamma_{b}} \sum_{N=-\infty}^{\infty} \delta(x-2 d N),
\end{aligned}
$$

which must be solved at all $x$ in the class of even and $2 d-$ periodic functions. A periodic function can be expanded into the Fourier series:

$$
\begin{aligned}
\check{f}(x) & =\frac{1}{2 d} \sum_{n=-\infty}^{\infty} e^{i k_{n} x} \check{f}\left(k_{n}\right), \quad k_{n}=\frac{\pi}{d} n, \\
\check{f}\left(k_{n}\right) & =\int_{-d}^{d} e^{-i k_{n} x} \check{f}(x) d x .
\end{aligned}
$$

After this transformation, we reproduce Eq. (17) with the only difference that the continuous wave vector $k$ is substituted by discrete $k_{n}$.

As in Sec. III the requirement that $\check{f}(x)$ is even, is automatically satisfied since the equation contains only $k_{n}^{2}$, while the $2 d$-periodicity is guaranteed since we consider $k_{n}$ defined by Eq. (43).

The equivalence to the previous equations allows us to directly use the results of Secs. II and III obtained for the infinite $d$. The rule is very simple: in the case of finite $d$, all the results of Secs. 1 and $\Pi$ for the Fourier harmonics are valid if we substitute $k$ by $k_{n}$. The realspace function can then be calculated with the help of Eq. (43).

For example, we consider the case of multidomain SF structure with the $\mathrm{F}$ layer of thickness $d$. For the case of positive chirality, instead of Eq. (37) inside of the domain $\left(|y|<a_{0}\right)$, we obtain

$$
f_{L}(x, y)=\frac{1}{2 d} \sum_{k_{n}} e^{i k_{n} x} f_{1}\left(a_{0}\right) \frac{\sinh \left(\kappa_{0} y\right)}{\sinh \theta_{0}}
$$

where $f_{1}\left(a_{0}\right)$ is given by Eq. (36).

The formula (44) can be drastically simplified in the limit when the $\mathrm{F}$ film is thin for the long-range component but thick for the short-range one (i.e., $k_{h} \gg 1 / d \gg$ $\left.Q, k_{\omega}\right)$. In this case, the main contribution is given by the $n=0$ harmonic, since otherwise $\kappa$ in the denominator of Eq. (36) become very large. Therefore, Eq. (44) yields

$$
\begin{aligned}
f_{L}(x, y)=-\left(\frac{i f_{S} \operatorname{sgn} \omega}{2 d \gamma_{b} k_{h}^{2}}\right) & \frac{Q}{\kappa_{Q} \operatorname{coth} \theta_{Q}+\kappa_{0} \operatorname{coth} \theta_{0}} \\
& \times\left.\frac{\sinh \left(\kappa_{0} y\right)}{\sinh \left(\kappa_{0} a_{0}\right)}\right|_{k_{n}=0},
\end{aligned}
$$

where we have used Eq. A.12. The $x$ dependence has vanished since the $\mathrm{F}$ layer is thin. 


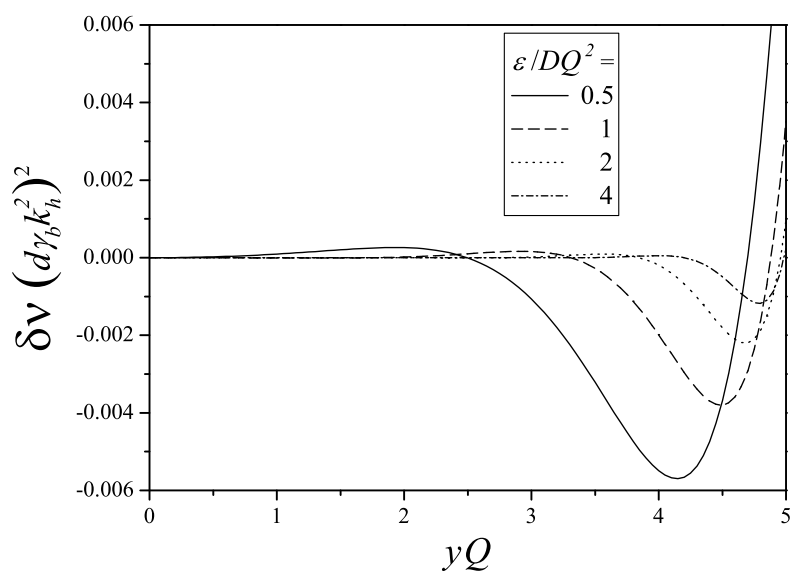

FIG. 2: Correction $\delta \nu(y)$ (due to the proximity effect) to the DOS at the free surface of the $\mathrm{F}$ layer in the case of positive chirality. $\delta \nu$ is multiplied by a dimensionless parameter $\left(d \gamma_{b} k_{h}^{2}\right)^{2}$, while $y$ is normalized by $Q$. The curves are plotted at several energies $\varepsilon$ (normalized by $D Q^{2}$ ). The width of the domains is $a_{0}=5 / Q$, while the rotation of magnetization in the domain walls corresponds to $Q a_{Q}=\pi$. We assume the limit $D Q^{2} \ll \Delta$, which means that the width of the domain walls is larger than the coherence length. At the boundary of applicability of our approximation, $\delta \nu(y)$ falls into experimentally measurable range ${ }^{41}$ (see text for details).

The variation of the DOS in space, $\delta \nu(y)=$ $-\operatorname{Re} f_{L}^{2} /\left.2\right|_{\omega \rightarrow-i \varepsilon}$, differs drastically from the case without the LRTC: it is almost constant across the layer (no $x$ dependence) and equal to zero in the middle of domains $(y=0)$. The condensate function $f_{L}(y)$ decays exponentially (oscillating at the same time) from the boundaries between domain walls and domains with characteristic length $\xi_{T}=\sqrt{D / 2 \pi T}$ (for the DOS, $\xi_{\varepsilon}=\sqrt{D / \varepsilon}$ ); see Fig. 22 for illustration. At the same time, the singlet component in the case of a domain structure leads to an even dependence of $\delta \nu(y)$ with respect to the middle of a domain. The characteristic length of the short-range components is $\xi_{h}=\sqrt{D / h}$. This case is realized if $\theta_{Q} \rightarrow 0$.

The case of negative chirality is treated similarly, and inside of the domain $\left(|y|<a_{0}\right)$ we obtain

$$
f_{L}(x, y)=\frac{1}{2 d} \sum_{k_{n}} e^{i k_{n} x} f_{1}\left(a_{0}\right) \frac{\cosh \left(\kappa_{0} y\right)}{\cosh \theta_{0}},
$$

where $f_{1}\left(a_{0}\right)$ is given by Eq. (40). In the limit $k_{h} \gg$ $1 / d \gg Q, k_{\omega}$, Eq. (46) yields

$$
\begin{aligned}
f_{L}(x, y)=-\left(\frac{i f_{S} \operatorname{sgn} \omega}{2 d \gamma_{b} k_{h}^{2}}\right) & \frac{Q}{\kappa_{Q} \operatorname{coth} \theta_{Q}+\kappa_{0} \tanh \theta_{0}} \\
& \times\left.\frac{\cosh \left(\kappa_{0} y\right)}{\cosh \left(\kappa_{0} a_{0}\right)}\right|_{k_{n}=0} .
\end{aligned}
$$

The resulting corrections to the DOS, $\delta \nu(y)$, in the case of positive and negative chiralities are compared in Fig. 3 .

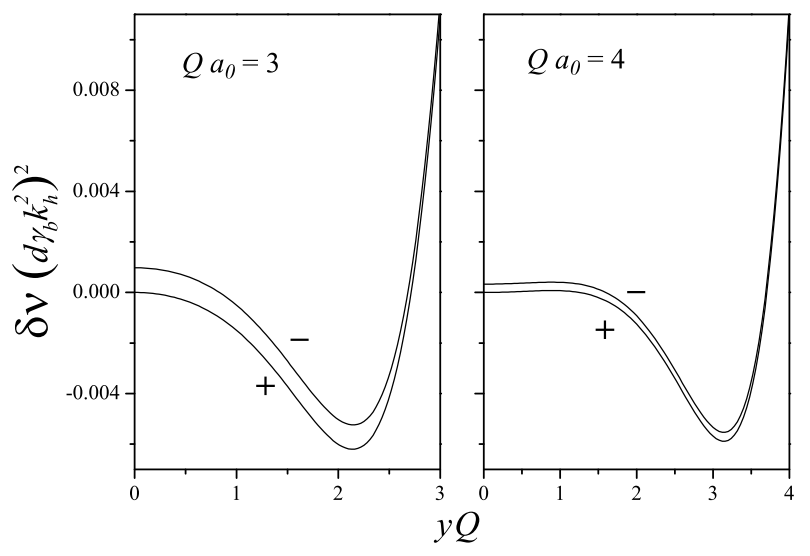

FIG. 3: Correction $\delta \nu(y)$ to the DOS at the free surface of the F layer: comparison of positive (denoted by "+") and negative (denoted by "-") chiralities at two widths of domains, $a_{0}=$ $3 / Q$ and $a_{0}=4 / Q$. The energy is $\varepsilon=0.5 D Q^{2}$. The difference between the chiralities becomes less pronounced as $a_{0}$ or $\varepsilon$ increases.

The dimensionless parameter $\left(d \gamma_{b} k_{h}^{2}\right)^{2}$, which multiplies $\delta \nu(y)$ in Figs. 2] and 3] is much larger than unity under our assumptions. This means that $\delta \nu(y)$ is very small. However, at the boundary of our approximation, when the parameter is of the order of unity, we expect no qualitative differences to appear. At the same time, in this case the DOS correction shown in Figs. 2 and 3 already falls into experimentally measurable range: in Ref. 41, the resolution of the DOS measurement was of the order $0.002 \div 0.003$. Experimentally, it is desirable to measure $\delta \nu$ at small energies, since the effect grows as energy decreases, see Fig. 2]

The behavior of the LRTC [and the corresponding correction to the DOS $\delta \nu(y)]$ inside of the domain walls is similar to Eqs. (44) and (46) with the main difference that the decay length in the $y$ direction is determined not by $\kappa_{0}$ but by $\kappa_{Q}$.

The coordinate dependence of the DOS presented in Figs. 2 and 3 differs from the corresponding dependence of the DOS variation caused by the singlet component. If the thickness of the $\mathrm{F}$ film $d$ is less than the exchange length $\xi_{h}$, then the DOS variation, related to the singlet component, in a domain wider than $\xi_{h}$, does not depend on $y$. In the case of the LRTC the DOS variation is a strongly coordinate-dependent quantity since the LRTC arises near the domain walls.

\section{CONCLUSIONS}

We have studied the long-range triplet superconductivity or LRTC in the SF multidomain structure, where magnetic domains are separated by the Néel domain walls. The magnetization vector $\mathbf{M}$ is supposed to lie in the plane of the F film. We show that in this case the LRTC arises at the domain walls and decays in do- 
mains over a large distance $\xi_{T}=\sqrt{D / 2 \pi T}$ (we assume the diffusive case). The same length characterize the decay of the LRTC from the SF interface. Although the amplitude of the LRTC is less than the amplitude of the singlet component in the $\mathrm{F}$ film at the interface by $k / k_{h}$ times, it decays much more slowly in the F film (here the characteristic value of the wave vector $k$ is of the order $\xi_{T}$ or $\left.d^{-1}\right)$. Therefore if the thickness of the $\mathrm{F}$ film $d$ is much larger than the "exchange" length $\xi_{h}=\sqrt{D / h}$, which characterizes the decay of the singlet component in the ferromagnet, only the LRTC $f$ survives at the outer surface of the F film in a SF structure. Its spatial in-plane dependence in domains $f(y)$ differs drastically from the corresponding dependence of the singlet component near the SF interface. If the vector $\mathbf{M}(y)$ rotates in the Néel walls in the same direction (positive chirality), then $f$ turns to zero in the centers of domains. This implies that the DOS variation due to the proximity effect $\delta \nu(\varepsilon, y) \sim f^{2}(\varepsilon, y)$ varies inside a domain turning to zero in the middle. Thus the measurements of the DOS variation at the outer surface of the F film in SF bilayers allows one to get an information on the nature of the condensate in the ferromagnetic films (singlet or triplet).

The effects of the LRTC are mostly pronounced at the boundaries between domains and domain walls. However, the correction to the DOS that we find is small since we assume the SF interface of low transparency and hence a weak proximity effect. At the same time, as we increase the interface transparency and reach the limit of applicability of our approximation, the correction to the DOS falls into experimentally measurable range, while we expect that our results are still qualitatively valid. Another way to increase the correction to the DOS is to measure it at smaller energies.

The obtained results provide an insight for the Josephson effect in SFS junctions with multidomain structure in the $\mathrm{F}$ layer. If the thickness of the $\mathrm{F}$ layer $d$ is much larger than the short exchange length $\xi_{h}$, the Josephson coupling between the S layers is due to the LRTC. In this case the local critical current density $j_{c}(y)$ is modulated in space, reaching its maxima at the domain walls and decaying to the centers of domains.

\section{Acknowledgments}

We are grateful to A. A. Golubov and M. V. Feigel'man for helpful discussions. We would like to thank SFB 491 for financial support. Ya.V.F. was also supported by the RFBR Grants Nos. 04-02-16348 and 04-02-08159, the RF Presidential Grant No. MK-3811.2005.2, the Russian Science Support Foundation, the Russian Ministry of Industry, Science and Technology, the program "Quantum Macrophysics" of the Russian Academy of Sciences, CRDF, and the Russian Ministry of Education.

APPENDIX: CALCULATING $A_{i l}$ AND $B_{i l}$

To find the coefficients $A_{i l}$ and $B_{i l}$, we match solutions (19) and (20) with the help of boundary conditions (11) and (12). This yields

$$
\begin{gathered}
A_{3+}+A_{3-}+F_{3}(Q)=B_{3+}+B_{3-}+F_{3}(0), \\
-A_{3+}+A_{3-}+F_{0}(Q)=-B_{3+}+B_{3-}+F_{0}(0), \\
-\frac{2 Q}{\kappa_{+}} A_{3+}+\frac{2 Q}{\kappa_{-}} A_{3-}+A_{1 L}=B_{1 L}, \\
-\kappa_{+} A_{3+}-\kappa_{-} A_{3-}=\kappa_{+} B_{3+}+\kappa_{-} B_{3-}, \\
\kappa_{+} A_{3+}-\kappa_{-} A_{3-}-Q B_{1 L}=-\kappa_{+} B_{3+}+\kappa_{-} B_{3-}, \\
Q\left[A_{3+}-A_{3-}+F_{0}(Q)\right]-\kappa_{Q} A_{1 L}=\kappa_{0} B_{1 L},
\end{gathered}
$$

where for brevity we have denoted

$$
\kappa_{Q} \equiv \kappa_{L}(Q), \quad \kappa_{0} \equiv \kappa_{L}(0) .
$$

From Eqs. A.4 and A.5 we find

$$
\begin{aligned}
& B_{3+}=-A_{3+}+\frac{Q}{2 \kappa_{+}} B_{1 L}, \\
& B_{3-}=-A_{3-}-\frac{Q}{2 \kappa_{-}} B_{1 L} .
\end{aligned}
$$

From Eqs. A.1 and (A.2) we find

$$
\begin{aligned}
2\left(A_{3+}+A_{3-}\right)+\frac{Q}{2}\left(\frac{1}{\kappa_{-}}-\frac{1}{\kappa_{+}}\right) B_{1 L}=-\delta F_{3}, \\
2\left(-A_{3+}+A_{3-}\right)+\frac{Q}{2}\left(\frac{1}{\kappa_{-}}+\frac{1}{\kappa_{+}}\right) B_{1 L}=-\delta F_{0},
\end{aligned}
$$

where $\delta F_{3}=F_{3}(Q)-F_{3}(0)$ and $\delta F_{0}=F_{0}(Q)-F_{0}(0)$. If Eq. (22) is fulfilled, on the order of magnitude we have $\delta F_{3} \propto\left(Q / k_{h}\right)^{2} F_{0}$ and $\delta F_{0} \propto\left(Q / k_{h}\right)^{2}\left(\left(k^{2}+2 k_{\omega}^{2}\right) / k_{h}^{2}\right) F_{0}$, where

$$
F_{0} \approx-\frac{i f_{S} \operatorname{sgn} \omega}{\gamma_{b} k_{h}^{2}}
$$

It follows from Eqs. A.10 and (A.11) that the coefficients $A_{3 \pm}$ are smaller than $B_{1 L}$ by the parameter $Q / k_{h}$.

From Eq. A.3 we find

$$
A_{1 L} \approx B_{1 L}
$$

From Eq. A.6 we find

$$
Q\left(A_{3+}-A_{3-}\right)-\kappa_{Q} A_{1 L}+Q F_{0}=\kappa_{0} B_{1 L} .
$$

The first term here is small, therefore we finally obtain

$$
A_{1 L} \approx B_{1 L} \approx \frac{Q F_{0}}{\kappa_{Q}+\kappa_{0}}
$$

These coefficients determine the amplitude of the LRTC in the domain $(y<0)$ and in the region with rotating magnetization $(y>0)$. 
* Electronic address: volkov@tp3.rub.de

$\dagger$ Electronic address: fominov@landau.ac.ru

1 A. I. Buzdin, Rev. Mod. Phys. 77, 935 (2005).

2 I. F. Lyuksyutov and V. L. Pokrovsky, Adv. Phys. 54, 67 (2005).

3 F. S. Bergeret, A. F. Volkov, and K. B. Efetov, cond-mat/0506047 Rev. Mod. Phys. (to be published).

4 P. G. de Gennes, Superconductivity of Metals and Alloys (Benjamin, New York, 1966).

5 F. S. Bergeret, A. F. Volkov, and K. B. Efetov, Phys. Rev. B 69, 174504 (2004); Europhys. Lett. 66, 111 (2004).

6 J. S. Jiang, D. Davidović, D. H. Reich, and C. L. Chien, Phys. Rev. Lett. 74, 314 (1995).

7 Th. Mühge, N. N. Garif'yanov, Yu. V. Goryunov, G. G. Khaliullin, L. R. Tagirov, K. Westerholt, I. A. Garifullin, and H. Zabel, Phys. Rev. Lett. 77, 1857 (1996); Th. Mühge, K. Theis-Bröhl, K. Westerholt, H. Zabel, N. N. Garif'yanov, Yu. V. Goryunov, I. A. Garifullin, and G. G. Khaliullin, Phys. Rev. B 57, 5071 (1998).

8 J. Aarts, J. M. E. Geers, E. Brück, A. A. Golubov, and R. Coehoorn, Phys. Rev. B 56, 2779 (1997).

9 L. Lazar, K. Westerholt, H. Zabel, L. R. Tagirov, Yu. V. Goryunov, N. N. Garif'yanov, and I. A. Garifullin, Phys. Rev. B 61, 3711 (2000).

10 I. A. Garifullin, D. A. Tikhonov, N. N. Garif'yanov, L. Lazar, Yu. V. Goryunov, S. Ya. Khlebnikov, L. R. Tagirov, K. Westerholt, and H. Zabel, Phys. Rev. B 66, 020505(R) (2002).

11 A. A. Golubov, M. Yu. Kupriyanov, and E. Il'ichev, Rev. Mod. Phys. 76, 411 (2004).

12 V. V. Ryazanov, V. A. Oboznov, A. Yu. Rusanov, A. V. Veretennikov, A. A. Golubov, and J. Aarts, Phys. Rev. Lett. 86, 2427 (2001).

13 T. Kontos, M. Aprili, J. Lesueur, F. Genêt, B. Stephanidis, and R. Boursier, Phys. Rev. Lett. 89, 137007 (2002).

14 Y. Blum, A. Tsukernik, M. Karpovski, and A. Palevski, Phys. Rev. Lett. 89, 187004 (2002).

15 A. Bauer, J. Bentner, M. Aprili, M. L. Della Rocca, M. Reinwald, W. Wegscheider, and C. Strunk, Phys. Rev. Lett. 92, 217001 (2004).

16 H. Sellier, C. Baraduc, F. Lefloch, and R. Calemczuk, Phys. Rev. Lett. 92, 257005 (2004).

17 F. S. Bergeret, A. F. Volkov, and K. B. Efetov, Phys. Rev. Lett. 86, 4096 (2001).

18 A. Kadigrobov, R. I. Shekhter, and M. Jonson, Europhys. Lett. 54, 394 (2001).

19 M. Eschrig, J. Kopu, J. C. Cuevas, and G. Schön, Phys.
Rev. Lett. 90, 137003 (2003).

20 A. F. Volkov, F. S. Bergeret, and K. B. Efetov, Phys. Rev. Lett. 90, 117006 (2003).

21 F. S. Bergeret, A. F. Volkov, and K. B. Efetov, Phys. Rev. B 68, 064513 (2003).

${ }^{22}$ Ya. V. Fominov, A. A. Golubov, and M. Yu. Kupriyanov, JETP Lett. 77, 510 (2003).

23 V. L. Berezinskii, JETP Lett. 20, 287 (1974).

24 T. R. Kirkpatrick and D. Belitz, Phys. Rev. Lett. 66, 1533 (1991); D. Belitz and T. R. Kirkpatrick, Phys. Rev. B 46, 8393 (1992); 60, 3485 (1999).

25 P. Coleman, E. Miranda, and A. Tsvelik, Phys. Rev. Lett. 70, 2960 (1993).

26 A. Balatsky and E. Abrahams, Phys. Rev. B 45, R13125 (1992); E. Abrahams, A. Balatsky, D. J. Scalapino, and J. R. Schrieffer, ibid. 52, 1271 (1995).

27 V. T. Petrashov , V. N. Antonov, S. V. Maksimov, and R. Sh. Shaikhaidarov, JETP Lett. 59, 551 (1994).

28 M. D. Lawrence and N. Giordano, J. Phys.: Condens. Matter 8, L563 (1996).

29 M. Giroud, H. Courtois, K. Hasselbach, D. Mailly, and B. Pannetier, Phys. Rev. B 58, R11872 (1998).

${ }^{30}$ V. T. Petrashov, I. A. Sosnin, I. Cox, A. Parsons, and C. Troadec, Phys. Rev. Lett. 83, 3281 (1999).

31 V. Peña, Z. Sefrioui, D. Arias, C. Leon, J. Santamaria, M. Varela, S. J. Pennycook, and J. L. Martinez, Phys. Rev. B 69, 224502 (2004).

32 P. Nugent, I. Sosnin, and V. T. Petrashov, J. Phys.: Condens. Matter 16, L509 (2004).

33 A. F. Volkov and A. Anishchanka, Phys. Rev. B 71, 024501 (2005).

34 F. S. Bergeret, A. F. Volkov, and K. B. Efetov, Phys. Rev. B 64, 134506 (2001).

35 Ya. M. Blanter and F. W. J. Hekking, Phys. Rev. B 69, 024525 (2004).

36 A. Aharoni, Introduction to the Theory of Ferromagnetism (Oxford, New York, 2001).

37 F. S. Bergeret, K. B. Efetov, and A. I. Larkin, Phys. Rev. B 62, 11872 (2000).

38 T. Champel and M. Eschrig, Phys. Rev. B 71, 220506(R) (2005); 72, 054523 (2005).

39 A. V. Zaitsev, Sov. Phys. JETP 59, 1015 (1984).

40 M. Yu. Kupriyanov and V. F. Lukichev, Sov. Phys. JETP 67, 1163 (1988).

41 T. Kontos, M. Aprili, J. Lesueur, and X. Grison, Phys. Rev. Lett. 86, 304 (2001). 